\title{
A Single Identified Interneuron Gates Tail-Shock Induced Inhibition in the Siphon Withdrawal Reflex Of Aplysia
}

\author{
William G. Wright ${ }^{1}$ and Thomas J. Carew ${ }^{2}$ \\ 'Department of Biology, Colorado State University, Fort Collins, Colorado 80523 and 'Departments of Psychology and \\ Biology, Yale University, New Haven, Connecticut 06520
}

\begin{abstract}
The marine mollusc Aplysia has proven very useful for a mechanistic analysis of behavioral modification. Among the stimuli used to modify the behavior of Aplysia, a noxious stimulus, tail shock, is one of the most effective. In addition to the extensively analyzed facilitatory effects of tail shock, recent work has demonstrated that it also produces marked transient inhibition in reflex responses. Here we report that functional removal (by hyperpolarization or voltage clamp) of a single inhibitory interneuron, L16, can eliminate most, if not all, of the inhibition in the siphon withdrawal reflex circuit produced by tail shock. In addition, this interneuron is strongly activated by tail shock. Finally, direct intracellular activation of $L 16$ does not, in itself, reliably produce inhibition, suggesting that L16 plays a gating role which is necessary for the expression of inhibition in the siphon withdrawal circuit. These results support the idea that behaviorally relevant neural modulation can be gated by a small number of neurons, in this case, by a single identified cell. Moreover, they indicate that in Aplysia, as in many other systems, the modulatory effects of a noxious stimulus are often funneled through a restricted neural locus before being distributed to the circuits actually responsible for generating the behavioral output.
\end{abstract}

[Key words: interneuron, neuronal network, abdominal ganglion, motor neuron, learning]

A complete understanding of the cellular mechanisms underlying experience-dependent modification of behavior requires elucidating not only the neural elements that are actually changed by an experience, but also the elements that are responsible for producing those changes. Recent work in a number of preparations suggests that, although the neural elements that are changed by experience are often widely distributed throughout the nervous system, the modulatory cells that induce the changes are often surprisingly localizcd (Marr, 1969; Albus, 1971; McCormick et al., 1985; Thompson, 1986; Mackey et al., 1987; Dudai, 1989; Mackey et al., 1989; Kapp et al., 1990; Davis et al., 1991; Davis, 1992; Small et al., 1992). These localized modulatory neurons typically have extensive projections which en-

\footnotetext{
Received March 29, 1993; revised June 28, 1994; acceptcd July 13, 1994.

We thank M. Davis, N. Emptage, T. Fischer, K. Fitzgerald, and E. Marcus for valuable comments on an earlier version of the manuscript. This work was supported by National Institute of Mental Health NRSA Grant 1-F32-MH09397 to W.G.W. and NSF Grant BNS 831 1300, NIH Grant R01-MH-14-1083, and AFOSR Award AF 89-0362 to T.J.C.

Correspondence should be addressed to Thomas J. Carew at the above address. Copyright (C) 1995 Society for Neuroscience $0270-6474 / 95 / 150790-08 \$ 05.00 / 0$
}

able them to exert widespread effects on diverse elements throughout the nervous system.

The siphon withdrawal reflex of Aplysia, which is well suited for a cellular analysis of behavior, exhibits a variety of forms of experience-dependent change. For example, noxious stimuli to the tail produce both short-term and long-term reflex facilitation (Walters et al., 1983; Hawkins et al., 1981 b; Scholtz and Byrne, 1987; Mercer et al., 1991). In addition, an opposite form of behavioral plasticity in this reflex has also been identified: tail shock, the same noxious stimulus that produces reflex facilitation, also produces transient reflex inhibition (KrontirisLitowitz and Walters, 1987; Mackey et al., 1987; Marcus et al., 1988). Recent work by Wright et al. (1991) indicates that the plasticity underlying this inhibitory modulation resides at least in part in the interneurons participating in the reflex.

In the present study, we have extended our cellular analysis of tail-shock induced inhibition in the siphon withdrawal reflex by examining the role of a single modulatory interneuron in tail-shock induced inhibition. We here show that the identified inhibitory interneuron, L16, is strongly activated by tail shock, as well as moderate to strong tactile stimuli to other sites on the body. We further show that L16's activity is required for the expression of tail-shock induced inhibition since reversibly blocking tail-shock induced activation of only this interneuron (by voltage clamp or hyperpolarization) virtually abolishes tailshock induced inhibition in the reflex circuit. Finally, we show that direct intracellular activation of L16, by itself, does not reliably produce inhibition in the siphon withdrawal circuit. This observation, taken together with the fact that L16-induced activity is necessary for the induction of inhibition, suggests that L16 plays a permissive role in the expression of the inhibitory process. Collectively, these results show that a single neuron can serve as a permissive "gate" in the expression of a basic form of behaviorally relevant reflex modulation in Aplysia.

A preliminary account of some of the results described in this article has been previously presented in abstract form (Wright and Carew, 1990).

\section{Materials and Methods}

Adult (75-125 gm) Aplysia californica were obtained from Marinus, Inc., $\mathrm{CA}$, and maintained at least $2 \mathrm{~d}$ in artificial sea water (Instant Ocean) at $15-17^{\circ} \mathrm{C}$ before an experiment. A reduced preparation similar to that used previously (Wright et al., 1991) was employed in all experiments (Fig. 1). Animals were anesthetized in ice-cold $\left(0.5-2.0^{\circ} \mathrm{C}\right)$ artificial seawater for 40-50 min prior to surgery. The tail and siphon (as well as the mantle and gill) were then surgically removed with their connections to the central nervous system left intact, and placed in a two-chambered Sylgard-lined plastic dish (Fig. 1). The central nervous system and siphon were pinned in one chamber. The tail was pinned 
in the other chamber, retaining its connection to the central nervous system (via P9 nerves) through a narrow slit between the chamber partition. The slit was then filled with Vaseline to electrically isolate the chambers, thus minimizing the electrical artifact produced in the physiological recordings during the electric shock to the tail.

In experiments in which we tested for the receptive field of $L 16$, the abdominal ganglion was withdrawn through an incision in the ventral surface of the animal with most of the peripheral nerves (e.g., siphon nerve and pleural abdominal connectives) intact. The body was then pinned in the recording chamber shown in Figure 1. Tests of the receptive field of L16 were performed by either tactile stimulation of various body regions with a Pasteur pipette, or by pinching these regions with forceps.

The ventral surface of the abdominal ganglion was surgically deshcathed, and a siphon motor neuron (LFS ; Frost et al., 1988) and interneuron L16 (Hawkins et al., 1981a,b) were impaled with glass microelectrodes (12-20 M 2 ) filled with $3 \mathrm{M} \mathrm{KCl}$. Siphon motor neurons $\left(\right.$ LFS $\left._{a}\right)$ were identified by position, size, input resistance $(40-100 \mathrm{M} \Omega$ ), and ability to produce head-directed (as opposed to tail-directed) siphon withdrawal when activated by intracellular current (Frost et al., 1988). L16 was identified by position and size as well as its synaptic connections (Hawkins et al., 1981a). In addition, we observed that L16 action potentials have a distinctively shaped afterhyperpolarization, with fast and slow components, which we found to be highly characteristic of L16. In some experiments a second, lower-resistance electrode (5-10 M $\Omega$ ) was inserted into L16 to pass hyperpolarizing current under current clamp, or to be used as the current-passing electrode when a two-electrode voltage-clamp mode was used (see below).

Watcr-jet stimulation of the siphon was performed with a Picospritzer (General Valve Corp.) that delivered constant-pressure pulses of sea water through a glass tube (inner diameter $=5 \mathrm{~mm}$ ). For each waterjet stimulus the maximum amplitude of the complex EPSP evoked in the siphon motor neuron was measured (the motor neuron was hyperpolarized $40-50 \mathrm{mV}$ from rest to prevent action potentials). Although water-jet stimuli usually produced a 5-10 mV EPSP in L16, they never elicited action potentials. Thus, in these experiments, L16 was not an active element in the siphon withdrawal circuit; therefore, its role in producing changes in the circuit was exclusively modulatory (see Discussion).

Experimental and control protocols were used to explore the effects of L/16 inactivation during tail shock (Fig. 2). Both protocols consisted of repeated phases. Each phase consisted of four water-jet stimuli delivered to the siphon at a nonhabituating $10 \mathrm{~min}$ interval (Wright et al., 1991). Ninety seconds before the third stimulus, an electric shock was delivered through bipolar capillary electrodes in contact with the dissected tail. The magnitude and duration of tail shock was identical to that used in previous experiments (four $1 \mathrm{sec}$ pulses of $50 \mathrm{~mA} \mathrm{AC}$ current; Marcus et al., 1988; Wright et al., 1991).

Experimental protocol. In the first phase of the experimental protocol, tail shock was delivered with L 16 at rest. If, in the first phase, tail shock did not induce at least $20 \%$ inhibition in the complex EPSP, the experiment was discontinued ( 2 of 11 experiments were discontinued for failing to meet this criterion). In the second phase, L16 was held 60$100 \mathrm{mV}$ below rest with either current or voltage clamp (using an Axoclamp voltage clamp in two-electrode mode) during the tail shock (see Fig. 2). Because repolarization of $\mathrm{L} 16$ produced rebound excitation and a barrage of action potentials, which could confound the $90 \mathrm{sec}$ test, we did not restore L16 to its resting potential until after the $90 \mathrm{sec}$ test. In the third phase, L16 was once again at rest during tail shock.

Control protocol. In order to control for refractoriness of the inhibitory process to repeated noxious stimulation, we performed a control procedure in which L 16 was left at rest in two successive phases. Again, if tail shock did not induce at least $20 \%$ inhibition in the complex EPSP during the first phase, the experiment was discontinued (one of eight experiments were discontinued for failing to meet this criterion).

To quantitatively assess inhibition within each phase, the amplitudes of the water-jet elicited EPSPs, $90 \mathrm{sec}$ and $10 \mathrm{~min}$ after tail shock, were standardized to the mean of the two preshock FPSPs, and compared to $100 \%$ (no change) with a $t$ test. The $90 \mathrm{sec}$ post shock tests from the different phases within a protocol were compared with a repeated measures $t$ test. Finally, the $90 \mathrm{sec}$ test of phase two in the experimental protocol was compared to the $90 \mathrm{sec}$ test of the control protocol with a two-sample $t$ test.

Direct L16 activation. Hawkins et al. (1981b) first demonstrated a modulatory role of L16 by showing that the complex EPSP in the gill

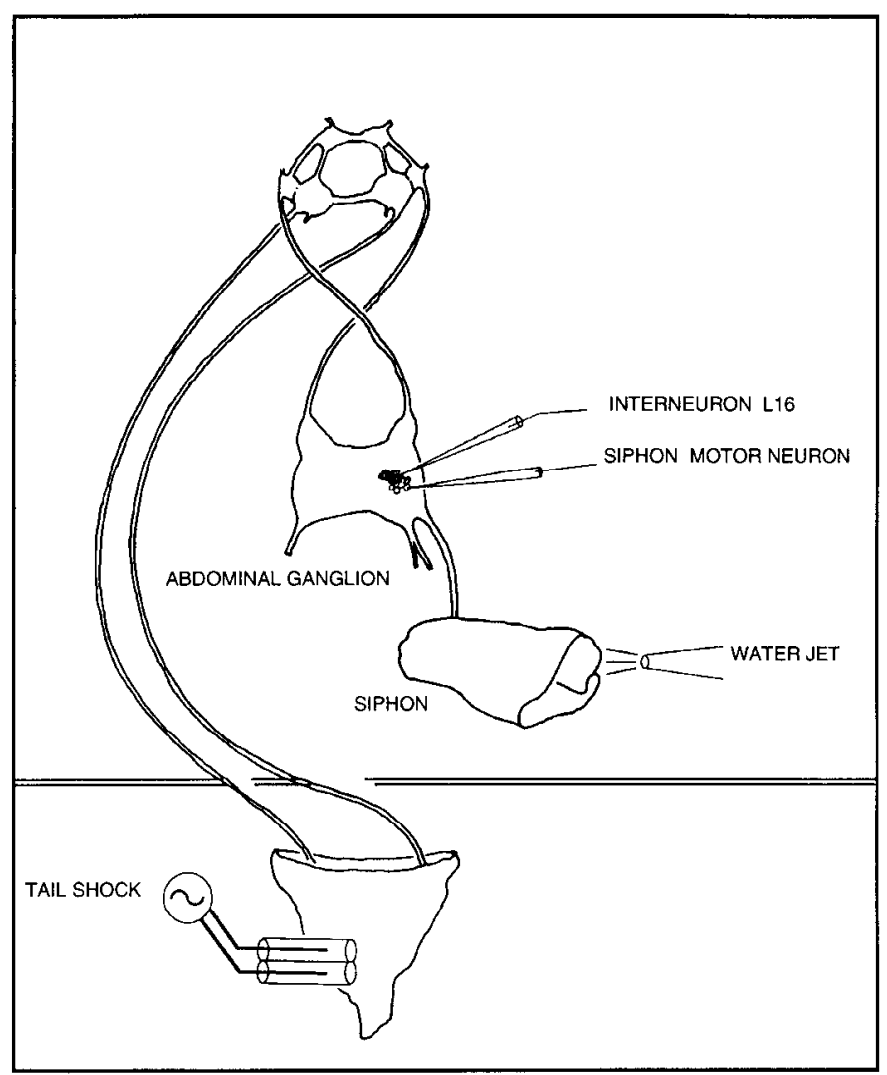

Figure 1. Schematic representation of the reduced preparation used to analyze the neuronal correlates of tail-shock induced reflex inhibition. The CNS is surgically removed, leaving it connected to the siphon and tail by the siphon nerve and $P 9$ nerves respectively. The tail is isolated in a separate chamber to allow delivery of electric shock without inducing electrical artifacts in the recording electrode. A water-jet stimulus to the siphon is used to elicit complex EPSPs in siphon motor neurons.

motor neuron $\mathrm{L} 7$ elicited by branchial nerve stimulation was inhibited for up to $40 \mathrm{sec}$ following intracellular activation of L16. We similarly tested whether L16 activation could inhibit the complex EPSP in siphon motor neurons in response to water-jet stimuli. Water-jet stimuli were delivered to the siphon at a $5 \mathrm{~min}$ interval and the height of the elicited complex EPSP in an LFS cell was recorded, as above. Ten to $20 \mathrm{sec}$ before the third water jet stimulus, L16 was activated by intracellular injection of four pulses of current (10-30 nA, 1-2 sec duration) which induced L16 to fire at $25-50 \mathrm{~Hz}$, producing a total of $100-200$ action potentials. This firing frequency was approximately equivalent to that recorded from L16 during tail shock. In order to test for L16-induced inhibition of the siphon withdrawal circuit, the height of the third EPSP (following L16 activation) was compared to that of the mean of the first two stimuli. Data were analyzed by means of a within-group $t$ test.

\section{Results}

Previous work (Wright et al., 1991) demonstrated that the reduced preparation used in the present study (Fig. 1) shows tailshock induced reflex inhibition similar to that observed in the intact animal (Marcus et al., 1988). Wright et al., (1991) further found that a neural correlate of reflex activation, the complex EPSP in siphon motor neurons elicited by water-jet stimuli to the siphon, was also inhibited $90 \mathrm{sec}$ after tail shock. Moreover, at the same time that inhibition was observed behaviorally and in the motor neuron complex EPSP, it was not reflected in the amplitude of the monosynaptic EPSP from siphon sensory to siphon motor neurons, suggesting that modulation of interneu- 


\section{EXPERIMENTAL}

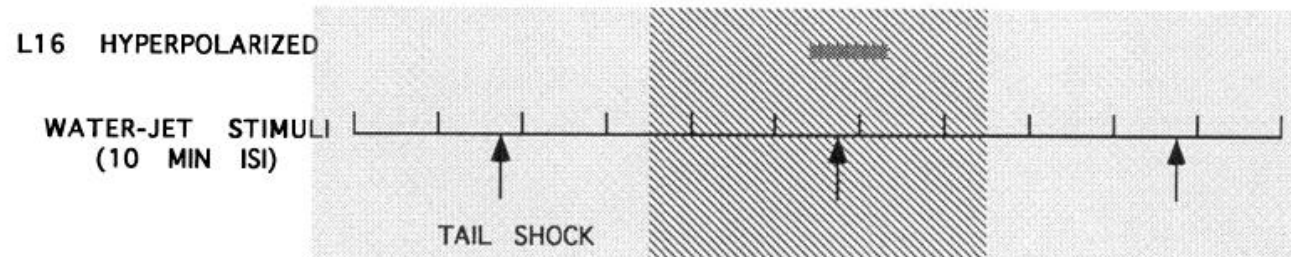

Figure 2. Two different protocols used to explore the role of interneuron L16 in tail-shock induced inhibition. In both protocols, siphon stimuli were delivered every 10 min (vertical tick marks); $90 \mathrm{sec}$ before the third stimulus a strong electric shock was delivered to the dissected tail (arrow). The experimental protocol (top) started with a control phase, in which the tail was shocked with L16 at rest. In the second phase of the experimental protocol L16 was hyperpolarized (shaded bar at top) during tail shock. In the third phase, tail shock was delivered with $\mathrm{L} 16$ once again at rest. The control protocol (bottom) consisted of two successive phases in which L16 was at rest during the tail shock.

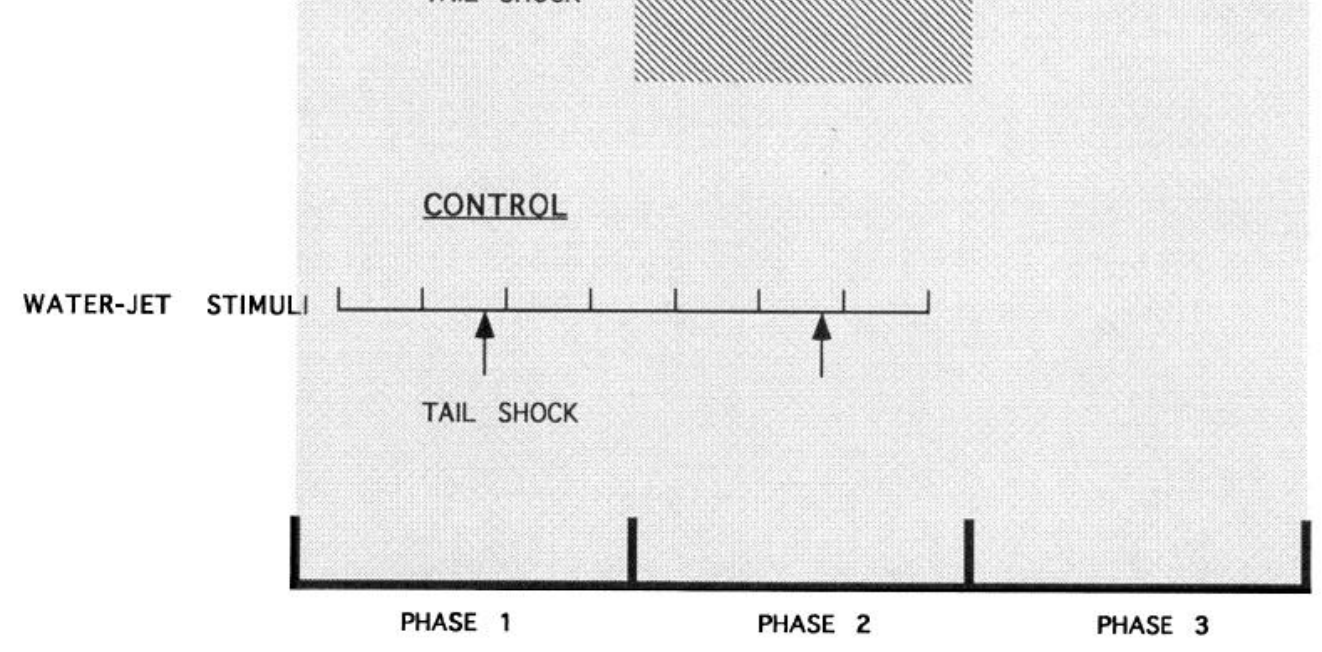

rons in the reflex pathway is at least partly responsible for the observed inhibition (Wright et al., 1991).

In the present study we explore the potential modulatory role of an identified inhibitory interneuron, L16, in tail-shock induced inhibition. This interneuron produces conventional fast IPSPs in a wide range of neurons within the siphon withdrawal circuit, including mechanosensory neurons (LE sensory neurons; Hawkins et al., 1981b), excitatory interneurons (L29 and L34; W. Frost and E. Kandel, personal communication), and motor neurons (LFS; Frost and Kandel, personal communication). Thus, L16 is synaptically connected to several important excitatory components of siphon withdrawal. Furthermore, Hawkins et al. (1981b) showed that direct intracellular activation of this interneuron can produce inhibition of the complex EPSP observed in the gill motor neuron L7 in response to electrical stimulation of the branchial nerve. These prior results led us to consider this cell as a potential locus through which modulatory stimuli might be gated to produce tail-shock induced inhibition of siphon withdrawal reflex circuitry.

\section{L16 is activated by tail shock, as well as tactile stimuli to other parts of the body}

If L16 is involved in tail-shock induced inhibitory modulation, L16 should be activated by tail shock. To examine this question, we recorded intracellularly from the interneuron during delivery of the same modulatory tail shock that was used in previous behavioral and cellular studies to induce behavioral inhibition (Marcus et al., 1988; Wright et al., 1991). L16 is normally silent, with relatively few spontaneous postsynaptic potentials, and no action potentials. The response of L16 to four successive $1 \mathrm{sec}$ shocks delivered to the dissected tail is shown in Figure 3. This pattern of L16 firing in response to a tail stimulus is typical: the first of the four shocks usually produces the greatest number and frequency of action potentials. Tail shock always induced
L16 to fire $(N=9)$; the response ranged from 20 to 120 action potentials (mean $=62$ ) with maximum firing frequencies as high as $50 \mathrm{~Hz}$. Thus, L16, a normally silent interneuron, is strongly activated by tail shock.

To examine whether tactile stimuli from other regions of the body also activate L16, we used a semireduced preparation consisting of the entire body of the animal, and recorded from L16 with the abdominal ganglion still connected to the body via the siphon nerve and pleural abdominal connectives (see Materials and Methods). Moderate tactile stimuli (manual stimulation with a glass pipette) delivered to the tail, rhinophore, oral tentacles, lateral body wall, and siphon elicited action potentials in L16. Stronger tactile stimuli (pinching with mousetooth forceps) to these same regions elicited stronger activity (5-10 spikes $\left.\mathrm{sec}^{-1}\right)$. The lack of response of L16 to water-jet stimuli (see Materials and Methods) was due to the relatively lower stimulus strength of the water jet. Thus, in addition to its activation by tail shock, L16 is also activated by moderate to strong tactile stimuli delivered to many other regions of the body.

\section{Inactivation of L16 reversibly blocks tail-shock induced inhibition}

If L16 has a primary modulatory role in tail-shock induced inhibition in the siphon withdrawal circuitry, then reversible inactivation of L16 by hyperpolarization or voltage clamp during tail shock should reduce or abolish the inhibition of the water-jet elicited complex EPSP $90 \mathrm{sec}$ after tail shock. To test this prediction, we examined the effects of tail-shock both with and without functional participation of L16 (Fig. 2). Specifically, in the first phase of the experimental protocol, four water-jet stimuli were delivered to the dissected siphon at a nondecrementing $10 \mathrm{~min}$ interval. Ninety seconds before the third stimulus, an electric shock was delivered to the dissected tail. Pre- 


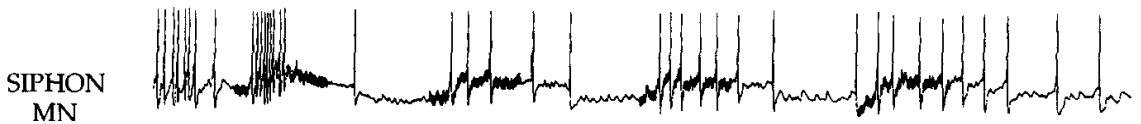

L16

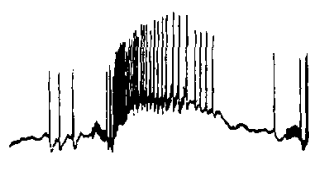

TAIL
SHOCK

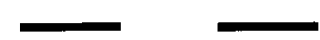

vious work demonstrated that tail-shock produces robust inhibition in the water-jet elicited complex EPSP at this first post-shock stimulus (Wright et al., 1991). In the second phase we repeated the experiment exactly, with the exception that L16 was functionally removed from the circuit by hyperpolarization (in current-clamp or voltage-clamp mode) during tail shock. Finally, in the third phase, L16 was once again allowed to fire in response to tail shock.

An example of our results is shown in Figure 4. Phase 1 is shown in the top traces: Confirming Wright et al. (1991), the complex EPSP elicited by water-jet stimulation is profoundly reduced $90 \mathrm{sec}$ after tail shock, and recovers $10 \mathrm{~min}$ later. In Phase 2, L16 is prevented from firing by voltage clamp during the tail shock and the inhibition observed in Phase 1 is completely abolished (Fig. 4, middle traces). Tail shock induced excitation to the motor neuron was unchanged during voltage clamp of L16 (not shown), indicating that inactivation of L16 does not change the efficacy of tail shock input to the siphon withdrawal circuitry. Finally, in Phase 3, with L16 again allowed to fire, the complex EPSP is again inhibited $90 \mathrm{sec}$ after tail shock and recovers $10 \mathrm{~min}$ later (Fig. 4, bottom traces).

A summary of nine experiments is shown in Figure 5. In Phase 1, significant inhibition of the complex EPSP was observed $90 \mathrm{sec}$ after tail shock $(p<0.001)$ and recovered $10 \mathrm{~min}$
Figure 3. Tail shock strongly activates interneuron L16. Simultaneous intracellular recordings from $\mathrm{L} 16$ and an identified siphon molor neuron (MN) are shown. Four $1 \mathrm{sec}$ A.C. electric shocks (indicated by horizontal bars) are delivered to the tail. L16 is normally silent. The EPSPs and action potentials in L16 just prior to the first shock are due to tactile stimulation caused by the capillary electrode contacting the tail (see Materials and Methods). Each tailshock elicits a brisk train of action potentials in L16 and moderate activation of the siphon motor neuron. later. In Phase 2, when L16 was inactivated during identical tail shock, there was no significant change from the preshock baseline at the $90 \mathrm{sec}$ test. In addition, the EPSP amplitude at the $90 \mathrm{sec}$ test of Phase 2 was significantly greater than that of the 90 sec test of Phase $1(p<0.001)$. Finally, in Phase 3, when L16 was no longer hyperpolarized, significant inhibition $(p<$ 0.02 ) followed by recovery was once again observed. A comparison of the mean EPSP of the $90 \mathrm{sec}$ test of Phase 3 with that of Phase 2, when L16 was hyperpolarized, was marginally significant ( $p=0.04$, one tail). These results show that silencing L16 during tail shock reversibly abolishes inhibitory modulation of the siphon withdrawal circuit.

During Phase 2 of these experiments, L16 was hyperpolarized not only during tail shock, but also during delivery of siphon stimulation, $90 \mathrm{sec}$ later (see Materials and Methods). This procedure was followed because of L16's strong tendency to fire action potentials upon release from hyperpolarization, which could potentially confound the measurement of inhibition. Our weak water-jet stimuli to the siphon do not produce action potentials in L16 (see Materials and Methods), so hyperpolarizing L16 would not have any conventional synaptic effect on other elements in the siphon withdrawal reflex circuitry. However, there remains a possibility that L16 is electrically coupled to other inhibitory elements in the reflex. If such were the case, AT REST

$L 16$ CLAMPED

L16 AT REST
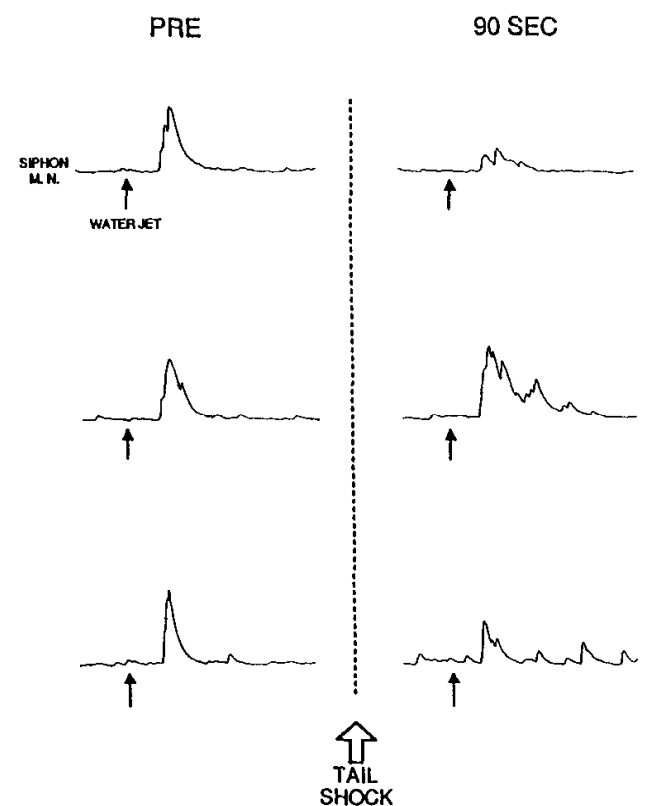

10 MIN
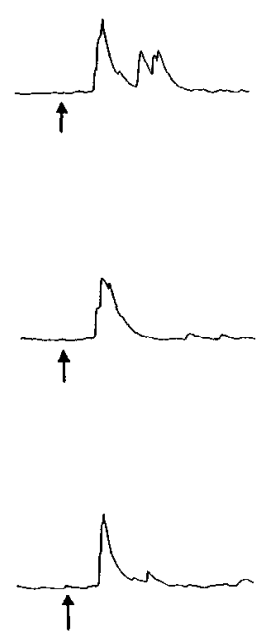

$\varlimsup_{\substack{200 \\ m s e c}}^{20}$
Figure 4. Inactivation of interneuron L16 during tail shock reversibly abolishes inhibition of the complex EPSP. Each row of traces shows the complex EPSP before (PRE), and $90 \mathrm{sec}$ and 10 min after an electric shock to the tail. In Phase I (top row of traces) tail-shock elicited inhibition in the complex EPSP is observed in the $90 \mathrm{sec}$ test, and recovers 10 min later. In Phase 2 (begun $50 \mathrm{~min}$ after the beginning of Phase I, see Fig. 2), the experiment is repeated, but with $\mathrm{L} 16$ inactivated by voltage clamp during tail shock. No inhibition of the complex EPSP is observed in the $90 \mathrm{sec}$ test. In Phase 3 (bottom row of traces) the experiment is repeated as in Phasc 1 and inhibition is again observed. 


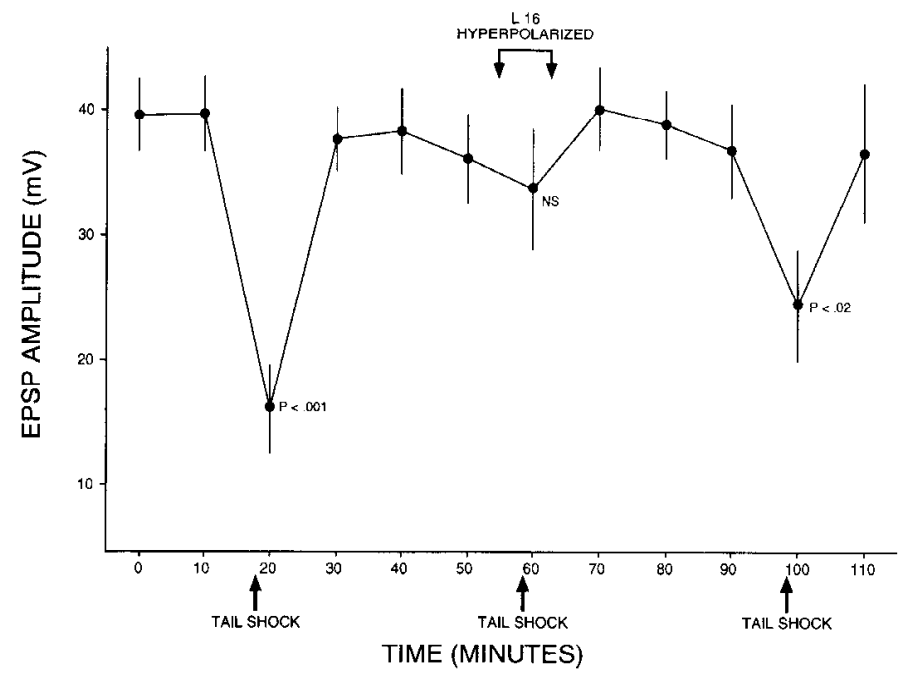

Figure 5. Summary of experiments in which interneuron L16 is inactivated during tail-shock. In Phase 1, when L16 is allowed to respond to tail-shock, significant $(p<0.001)$ inhibition of the complex EPSP is observed $(N=9)$. In Phase 2 , the inhibition is abolished when L16 is inactivated (hyperpolarized) during tail-shock. In Phase 3 , when L16 is once again allowed to fire, significant $(p<0.02)$ inhibition is once again produced by tail-shock. The post-shock complex EPSP is significantly greater when L16 is hyperpolarized during tail shock than the prior post-shock EPSP when L16 is at rest during the tail shock $(p<0.001)$. In addition, the complex EPSP shortly after the third tail shock, when L16 is again at rest is marginally more inhibited $(p=0.076)$ than when L16 is hyperpolarized. In this and subsequent figures, the data are expressed as means \pm SEMs.

hyperpolarization of $\mathrm{L} 16$ could, in principle, reduce the contribution of such inhibitory elements to the complex EPSP, thereby attenuating inhibition in the reflex circuit that would normally occur in response to water-jet stimulation of the siphon. Thus the lack of inhibition observed in the $90 \mathrm{sec}$ post-shock test of Phase 2 (Figs. 4, 5) could be due to the removal of an inhibitory clcment of the reflex itsclf, rather than to the removal of external inhibitory modulation of the reflex. This hypothesis would predict that hyperpolarizing L16 in control conditions should, by itself, result in a larger water-jet elicited EPSP (since other putative inhibitory elements electrically coupled to L 16 would be silenced during siphon stimulation). To directly test this hypothesis we performed six experiments (three preparations) to investigate whether the hyperpolarization of L16 per se would alter the amplitude of the water-jet elicited EPSP. We used a reduced preparation consisting of the siphon and central nervous system, and stimulated the siphon with water-jet stimuli three times at a 5 min interval while recording from L16 and LFS cells. Just prior to the third stimulus, L16 was hyperpolarized in the same way as in the above experiments. Hyperpolarization of L16 had no effect on the amplitude of the complex EPSP ( $n$ $=3$; mean control EPSP: $35.2 \pm 2.6 \mathrm{mV}$; mean EPSP with L16 hyperpolarized: $35.3 \pm 3.5 \mathrm{mV}$ ). Thus the reduction of inhibition observed in Phase 2 of the experiments above, which was induced by hyperpolarizing $\mathrm{L} 16$ during tail shock and the subsequent 90 sec test, cannot be attributed to any action of L16 during the test itself.

In the experiments shown in Figures 4 and 5 , it is possible that at least some of the reduction of inhibition during L16 inactivation (Phase 2) was due to refractoriness of the inhibitory process. To directly examine this possibility, we performed 7

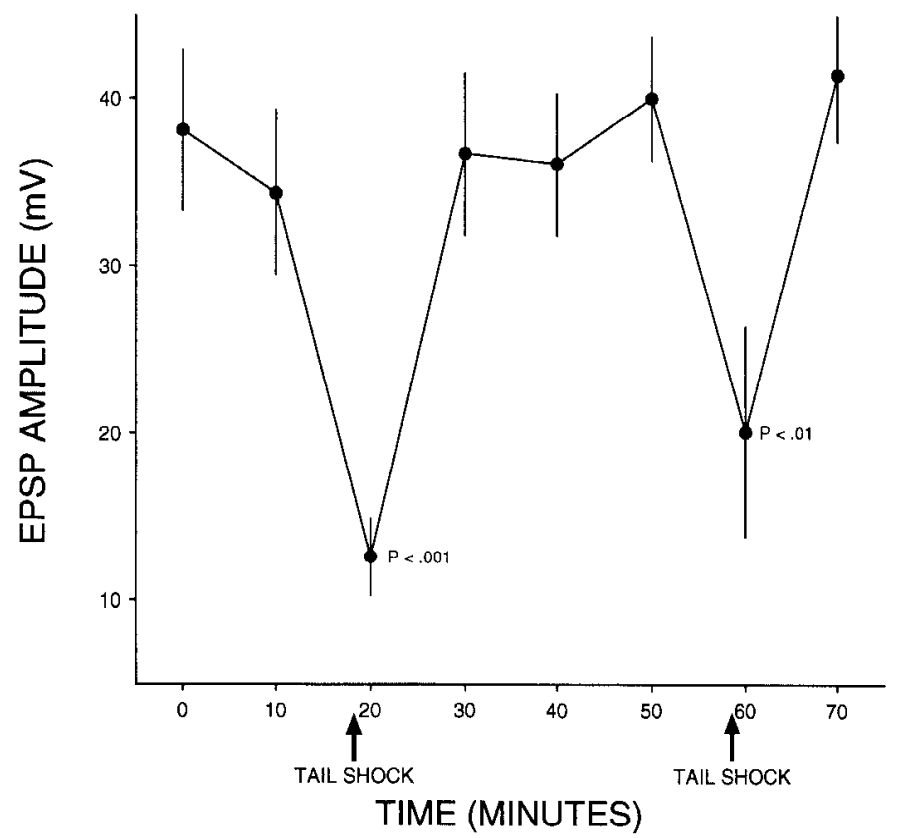

Figure 6. Summary of control experiments. When L16 is allowed to fire in two sequential presentations of tail-shock, significant inhibition of the complex EPSP is observed $90 \mathrm{sec}$ after both presentations $(N=$ 7). Thus, the inhibitory process shows no significant refractoriness when repeatedly activated.

additional control experiments identical to the experiments summarized in Figure 5, except that L16 was allowed to fire in both Phases 1 and 2 (Fig. 2). These results are summarized in Figure 6, and confirm that each of two successive applications of tail shock produced significant inhibition in the siphon withdrawal reflex circuitry. A direct comparison of the $90 \mathrm{sec}$ test experiments in which L16 is inactivated in Phase 2 (Fig. 5) with the control experiments in which L16 is allowed to fire in Phase 2 (Fig. 6), reveals that inactivation of $\mathrm{L} 16$ produces a significant rcduction $(p<0.04)$ in tail-shock induced inhibition (Fig. 7). Thus refractoriness of the inhibitory process with repeated tailshock cannot account for the reduction of inhibition when L16 is inactivated. Rather, our results support the conclusion that participation of L16 is necessary for the expression of tail-shock induced inhibition.

\section{Activation of $L 16$ alone does not consistently produce inhibition of siphon withdrawal circuitry}

If $\mathrm{L} 16$ is a source of inhibitory modulation, its direct activation alone should produce inhibition in the neural circuit mediating siphon withdrawal. Hawkins et al. (1981b) addressed this question in the gill withdrawal circuit by using electrical stimulation of the branchial nerve to induce a complex EPSP in gill motor neuron $\mathrm{L} 7$. They found that activation of $\mathrm{L} 16$ with intracellular depolarizing current prior to stimulation of the branchial nerve reversibly reduced the amplitude of the ncrve-evoked complex EPSP.

To ask whether L16 is a source of inhibitory modulation under our experimental conditions (using natural water jet stimuli to elicit activity in siphon motor neurons), we examined the effects of intracellular activation of L16 on water-jet elicited complex EPSPs. In two of nine preparations, L16 activation inhibited the water-jet elicited complex EPSP in LFS motor neurons. However, an analysis of the entire data set showed 


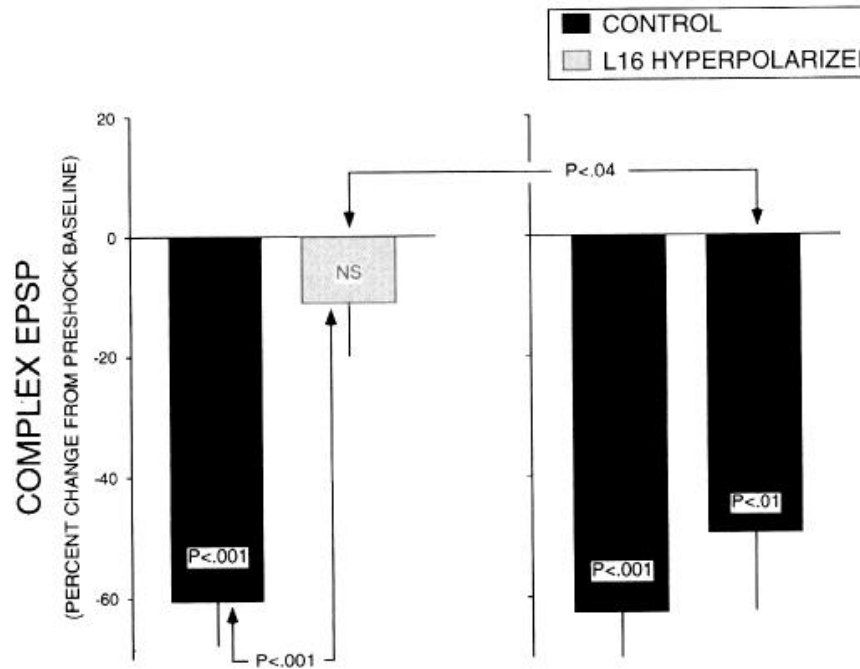

Figure 7. Summary analysis. The data from Figures 5 and 6 are summarized in the left and right histograms, respectively. EPSP amplitude at the $90 \mathrm{sec}$ post-shock test is expressed as percentage change from baseline. In control experiments when L16 is allowed to respond to tail shock (at right), inhibition is significantly greater than in experiments when L16 is inactivated by hyperpolarization (at left).

only a modest trend toward L16-induced inhibition, with no statistically reliable inhibition of the complex EPSP by prior activation of L16 (mean percent PRE: $87.5 \pm 7.5$; NS; Fig. 8). Thus these results indicate that $\mathrm{L} 16$, rather than producing inhibition itself, may act as a permissive gate for the expression of tail-shock induced inhibitory modulation in the siphon reflex circuitry.

\section{Discussion}

Functional role of the identified inhibitory interneuron $L 16$ The results of our experiments suggest that the inhibitory interneuron $\mathrm{L} 16$ has a pivotal role in gating tail-shock induced inhibition of the siphon withdrawal reflex. L16 is strongly activated by tail shock, and its activity is required for tail-shock induced inhibition of water-jet elicited EPSPs in siphon motor neurons. Interestingly, despite clear evidence of its central role in gating inhibition, L16's activation alone does not reliably produce inhibition of the siphon withdrawal circuitry. How can hyperpolarization of L16 during tail shock eliminate inhibition, when L16's activation alone does not produce inhibition? There are several possibilities. For example, it could be that L16 must be coactivated with other neurons in order to produce longlasting inhibition. There is precedence for such activity-dependent inhibition: Small et al. (1989) observed that pairing application of FMRFamide, a peptide that causes presynaptic inhibition of LE sensory neurons, with activity in those sensory neurons produces more inhibition of transmitter release than application of FMRFamide onto silent sensory neurons. Since LE siphon sensory neurons do not fire in response to tail shock, the locus of such activity-dependent inhibition would have to lie elsewhere in reflex or modulatory circuits. Another possibility is that L16 may make a rectifying electrical connection to one or more additional inhibitory elements. In such a case, hyperpolarization of L16 would hyperpolarize the postulated elements that give rise to inhibitory modulation, whereas depolarization and action potentials in L16 would have little effect on the hypothesized elements. These considerations highlight

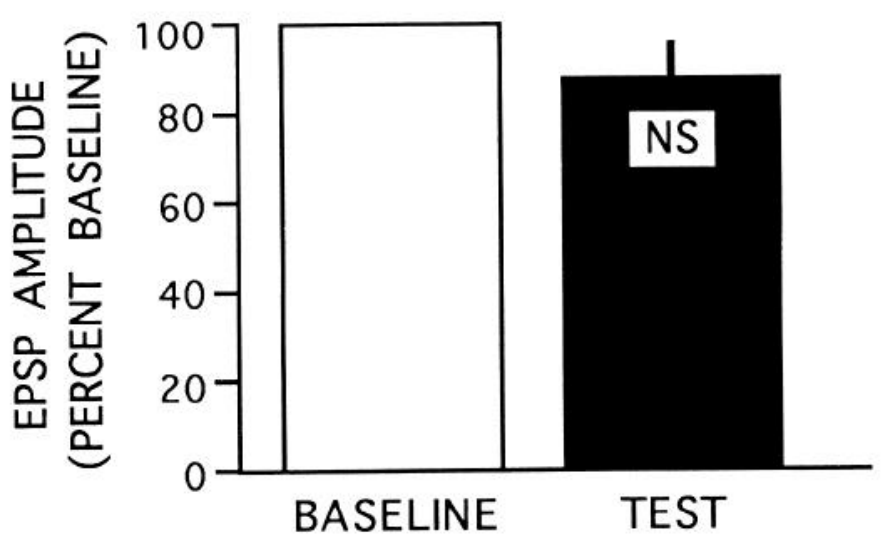

Figure 8. Direct activation of L16 does not significantly inhibit the water-jet elicited complex EPSP. The siphon was stimulated with a water jet every $5 \mathrm{~min}$. Fifteen seconds prior to the third water-jet stimulus, L16 was activated by intracellular injection of depolarizing current. Shown are mean \pm SEM from nine experiments. Although there is a modest trend towards inhibition, activation of L16 prior to siphon stimulation does not produce significant inhibition $(N=9 ; t=1.67 ; p$ $=0.13$ ).

the fact that L16 might be exerting its modulatory actions in concert with other inhibitory loci. The fact that very modest (and statistically insignificant) inhibition was observed when L16 was hyperpolarized (Fig. 5) may reflect the muted contribution of these other inhibitory elements in the absence of L16 activity. Thus L16 provides an interesting example of a unique interneuron that not only makes conventional fast IPSPs onto elements throughout a reflex circuit, but also serves as a permissive gate in the expression of a widespread inhibitory process.

L16 was first identified as an inhibitory interneuron by Hawkins et al. (1981a,b) who showed that activating this neuron with intracellular current could transiently reduce the amplitude of the branchial nerve-elicited complex EPSP in the gill motor neuron, L7. In the present study, we were unable to show a similar inhibition in the siphon withdrawal circuit. It is not clear whether the difference in the two results can be attributed to the difference in the two behavioral circuits (Gill withdrawal vs siphon withdrawal), or to the manner in which the circuits were activated (branchial nerve shock vs water-jet stimuli to the siphon). It will thus be of considerable interest to determine the experimental (and perhaps behaviorally relevant) contexts within which differences in the effects of direct activation of L16 are observed.

Segal and Koester (1982) provided evidence that the neurotransmitter used by L16 is acetylcholine. More recently, Trudeau and Castellucci (1992) showed that blocking cholinergic synapses in the CNS of Aplysia with curare increases the amplitude of the complex EPSP elicited in motor neurons by nerve shock. Thus, other inhibitory interneurons apparently more directly involved with mediation of siphon withdrawal, also appear to be cholinergic. Although curare may also block synapses from noncholinergic neurons (see Carpenter et al., 1976), these collective observations suggest that inhibitory cholinergic interneurons may play important mediating and modulating roles during reflex behaviors in Aplysia.

\section{The neural architecture of behavioral modulation}

These results address the question of the organization of the neural elements involved in experience-dependent modification 
of behavior. In particular, they support the idea that modulatory stimuli are often funnelled through a relatively small number of neurons (in the case of the present study a single neuron), which then relay the modulatory signal throughout a behavioral circuit. In contrast, the loci in the behavioral circuit that are changed by this modulatory signal are often distributed and widespread.

Evidence for this mode of neural control comes from a variety of vertebrate and invertebrate preparations, and is especially strong for learning paradigms in which aversive stimuli are used to effect behavioral modification. For example, in fear potentiated startle in the rat, a relatively restricted anatomical locus, the central nucleus of the amygdala, has been shown to be critical in translating aversive stimuli into circuit changes that result in a heightened startle response to auditory stimuli (Davis et al., 1991; Davis, 1992). Other behavioral manifestations of "fear" in a wide variety of response systems are likewise produced by activation of the amygdala (Kapp et al., 1984; Kapp et al., 1990; Davis, 1992). Thus, a relatively restricted locus can determine whether any given stimulus is "noxious" enough to change widely distributed behavioral circuits into the "fearful" mode.

Another example of a restricted modulatory locus is the inferior olivary complex in the rabbit, which plays a critical role in classical conditioning of the eyeblink response (McCormick et al., 1985; Thompson, 1986). Lesions restricted to this small nucleus abolish the ability of paired presentation of a tone (CS) with a corneal air puff (US) to produce a conditioned eyeblink response. Similarly, if animals are conditioned prior to lesion of the inferior olivary complex, following the lesion their conditioned eyeblink reflex shows extinction to paired tone and airpuff stimuli that is indistinguishable from that of animals given only the tone stimulus (see also Steinmetz and Thompson, 1991).

Several studies in Aplysia add further support to the hypothesis that a relatively small number of modulatory neurons produce diverse circuit modifications which underlie behavioral change following aversive stimuli. For example, serotoninergic facilitation is known to contribute to behavioral sensitization of the gill and siphon withdrawal reflex as well as the tail withdrawal reflex (Walters et al., 1983; Glanzman et al., 1989; Mackey et al., 1989; Mercer et al., 1991). Recently, a single pair of serotoninergic interneurons, the CB1 cells, were shown to be activated by tail shock and produce facilitation of the excitatory synaptic connection between siphon sensory neurons and their follower neurons (Mackey et al., 1989). Although other serotoninergic cells are present in the abdominal ganglion, and could, in principal, be participating in tail-shock induced facilitation, there is both anatomical and physiological evidence that the most important serotoninergic facilitators are outside the abdominal in the ring ganglia (Hawkins, 1989). Since there are only three major serotoninergic axons from the ring ganglia (two are from each of the two CB1s; Longley and Longley, 1986; Mackey et al., 1989; W.G.W., unpublished observations) entering each hemiganglion of the abdominal ganglion, it is reasonable to suppose that the CB1 cells act as a restricted locus gating 5-HT-mediated heterosynaptic facilitation of the mechanosensory neurons of the abdominal ganglion (Mackey et al., 1989). Although there are other nonserotoninergic interneurons in the abdominal ganglion that are also sensitive to tail shock, and produce facilitation of sensory input to the gill and siphon withdrawal reflex (Hawkins et al., 1981a,b), these modulators do not appear to be as important as serotoninergic cells, since selective poisoning of serotoninergic cells abolishes tail- shock induced heterosynaptic facilitation as well as sensitization (Glanzman et al., 1989). Thus, a single pair of facilitatory interneurons appears to be an important modulatory component contributing to tail-shock induced sensitization of the siphon withdrawal reflex.

In addition to facilitatory effects, inhibitory modulation can also be produced by relatively few neurons in Aplysia. For example, activation of the L29 interneuron, in addition to producing facilitatory effects, also produces pronounced recurrent inhibition in the siphon withdrawal circuitry (Fischer and $\mathrm{Ca}$ rew, 1991, 1993). In addition, the FMRFamide containing neuron, LP1 16, is activated by tail shock and produces short-lived presynaptic inhibition of sensory to motor neuron synapses (Mackey et al., 1987; Small et al., 1992). It should be noted that this inhibition is too short lived $(30 \mathrm{sec})$ to contribute to the tail-shock induced inhibition of siphon withdrawal reflex (tested $90 \mathrm{sec}$ post-shock) in the present study.

Thus, noxious stimuli such as tail shock or connective stimulation appear to act through a small number of identified modulatory neurons, which in turn modulate a wide variety of neurons that are involved with the siphon and gill withdrawal reflex. This prior research has demonstrated that identified modulatory cells are sufficient to effect widespread changes in reflex circuitry. However, the possibility that an individual neuron is necessary for a particular form of modulation is more difficult to demonstrate. We have provided such evidence for cell L16 in gating tail-shock induced inhibition of the siphon withdrawal reflex. This demonstration further strengthens the hypothesis that a restricted neural locus can have far reaching modulatory effects on the neural circuits underlying behavior.

\section{References}

Albus JS (1971) A theory of cerebellar function. Math Biosci 10:2561.

Carpenter DO, Swann JW, Yarowsky PJ (1976) Effect of curare on responses to different putative neurotransmitters in Aplysia neurons. J Neurobiol 8:119-132.

Davis M (1992) The role of the amygdala in fear and anxiety. Annu Rev Neurosci 15:353.

Davis M, Hitchcock JM, Rosen JB (1991) Neural mechanisms of fear conditioning measured with the acoustic startle reflex. In: Neurobiology of learning emotion and affect (Madden JI, ed). New York: Raven.

Dudai Y (1989) The neurobiology of memory. New York: Oxford UP.

Fischer T, Carew TJ (1991) Activation of the facilitatory interneuron L29 produces inhibition of reflex input to siphon motor neurons in Aplysia. Soc Neurosci Abstr 17:1302.

Fischer T, Carew TJ (1993) Activity-dependent potentiation of recurrent inhibition: a mechanism for dynamic gain control in the siphon withdrawal reflex of Aplysia. J Neurosci 13:1302-1314.

Frost WN, Clark GA, Kandel ER (1988) Parallel processing of shortterm memory for sensitization in Aplysia. J Neurobiol 19:297-334.

Glanzman DL, Mackey SL, Hawkins RD, Dyke AM, I loyd PE, Kandel ER (1989) Depletion of serotonin in the nervous system of Aplysia reduces the behavioral enhancement of gill-withdrawal as well as the heterosynaptic facilitation produced by tail shock. J Neurosci 9:42004213.

Hawkins RD (1989) Localization of potential serotoninergic facilitator neurons in Aplysia by glyoxylic acid histofluorescence combined with retrograde fluorescent labeling. J Neurosci 9:4214-4226.

Hawkins RD, Castellucci VF, Kandel ER (1981a) Interneurons involved in mediation and modulation of gill-withdrawal reflex in Aplysia. I. Identification and characterization. J Neurophysiol 45:304 314.

Hawkins RD, Castellucci VF, Kandel ER (1981b) Interneurons involved in mediation and modulation of gill-withdrawal reflex in Aply- 
sia. II. Identified neurons produce heterosynaptic facilitation contributing to behavioral sensitization. J Neurophysiol 45:315-326.

Kapp BS, Pascoe JP, Bixler MA (1984) The amygdala: a neuroanatomical systems approach to its contribution to aversive conditioning. In: The neuropsychology of memory (Butters N, Squire LS, eds), pp 473-488. New York: Guilford.

Kapp BS, Wilson A, Pascoe JP, Supple WF, Whalen PJ (1990) A neuroanatomical systems analysis of conditioned bradycardia in the rabbit. In: Neurocomputation and learning: foundations of adaptive networks (Gabriel M, Moore J, eds). New York: Bradford.

Krontiris-Litowitz JK, MTE, Walters ET (1987) Central suppression of defensive reflexes in Aplysia by noxious stimulation and by factors released from the body wall. Soc Neurosci Abstr 13:815.

Longley RD, Longley AJ (1986) Serotonin immunoreactivity of neurons in the gastropod Aplysia californica. J Neurobiol 17:349-358.

Mackey SL, Glanzman DL, Small SA, Dyke AM, Kandel ER, Hawkins RD (1987) Aversive stimuli produce inhibition as well as sensitization of the siphon withdrawal reflex of Aplysia: a possible behavioral role for presynaptic inhibition mediated by the peptide FMRFamide. Proc Natl Acad Sci USA 84:8730-8734.

Mackey SL, Kandel ER, Hawkins RD (1989) Identified serotoninergic interneurons LCB1 and RCB1 in the cerebral ganglia of Aplysia produce presynaptic facilitation of siphon sensory neurons. J Neurosci 9:4227-4235.

Marcus EA, Nolen TG, Rankin CH, Carew TJ (1988) Behavioral dissociation of dishabituation, sensitization and inhibition in Aplysia. Science, 241:210-213.

Marr D (1969) A theory of cerebellar cortex. J Physiol (Lond) 202: $437-470$.

McCormick DA, Steinmetz JE, Thompson RF (1985) Lesions of the inferior olivary complex cause extinction of the classically conditioned eye blink response. Brain Res 359:120-130.

Mercer AR, Emptage NJ, Carew TJ (1991) Pharmacological disso- ciation of modulatory effects of serotonin in Aplysia sensory neurons. Science 254:1811-1813.

Scholtz KP, Byrne JH (1987) Long-term sensitization in Aplysia: biophysical correlates in tail sensory neurons. Science 235:685-687.

Segal MM, Koester J (1982) Convergent cholinergic neurons produce similar postsynaptic actions in Aplysia: implications for neural organization. J Ncurophysiol 47:742-759.

Small SA, Kandel ER, Hawkins RD (1989) Activity-dependent enhancement of presynaptic inhibition in Aplysia sensory neurons. Science 243:1603-1606.

Small SA, Cohen TE, Kandel ER, Hawkins RD (1992) Identified FMRFamide-immunoreactive neuron LPL16 in the left pleural ganglion of Aplysia produces presynaptic inhibition of siphon sensory neurons. J Neurosci 12:1616-1627.

Steinmetz JE, Thompson RF (1991) Brain substrates of aversive classical conditioning. In: Neurobiology of learning, emotion, and affect (Madden JI, ed), pp 97-120. New York: Raven.

Thompson RF (1986) The neurobiology of learning and memory. Science 233:941-947.

Trudeau L-E, Castellucci VF (1992) Functional uncoupling of cholinergic inhibitory interneurons plays an important role in short-term sensitization of Aplysia gill and siphon withdrawal reflex (GSWR). Soc Neurosci Abstr 18:531.

Walters ET, Byrne JH, Carew TJ, Kandel ER (1983) Mechanoafferent neurons innervating the tail of Aplysia. II. Modulation by sensitizing stimuli. J Neurophysiol 50:1543-1559.

Wright WG, Carew TJ (1990) Contributions of interneurons to tailshock induced inhibition of the siphon withdrawal reflex in Aplysia. Soc Neurosci Abstr 16:20.

Wright WG, Marcus EA, Carew TJ (1991) A cellular analysis of inhibition in the siphon withdrawal reflex of Aplysia. J Neurosci 11: 2498-2509. 